\title{
DISEÑO DE UN MODELO PARA LA MEDICIÓN DEL CAPITAL INTELECTUAL DE EMPRESAS DEMANUfaCtuRA EN
}

\section{RESUMEN}

EIModeloparalamedición del capital intelectual de unaempresademanufacturaenel Perútieneun valorimportantepor ser el elemento clave para conocerlaestructuradelosactivosintangibles dela empresaysucapacidad paragenerarvalor. La creación delvalorno sólo depende delosactivos físicos, sino cada vez más de los activosintangibles, por elloesque losindicadores del capita intelectual seestáidentificandoyestructurando paraqueel modelogaranticequelosresultadosquese obtengan sean aceptablesy creibles.

Palabras Clave: Medición del capital intelectual. Gestión del capital intelectual. Diseño del modelo de medición.

THE DESIGN OF A MODEL FOR THE INTELLECTUALCAPITAL MEASUREMENTIN MANUACTURING COMPANIES IN Peru ABSTRACT

The Modelfor the intellectual capital measurementin amanufacturing companyin Peruhasanimportant valueforbeing the keyelement to knowthestructure of the intangible assets of the company and its capability to generatevalue. The creation of value doesnotdependon physicalassetsonly, but increasingly on intangible assets. That is why the indicators of theintellectual capitalare beingidentified and built, so that the model guarantees, that the results to be obtained are acceptableand credible.

Key words: Intellectual capital measurement. Intellectual capital management. Design of the measurement model.

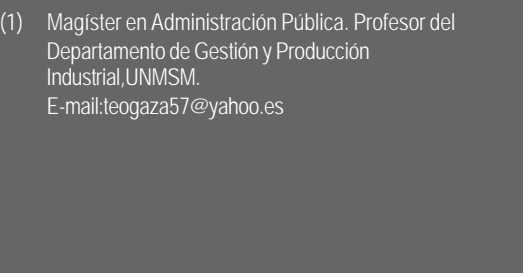

\section{INTRODUCCIÓN}

En nuestro país, la medición del Capital Intelectual aún no ha sido tomada en cuenta en los estados contables o en las Oficinas de Recursos Humanos de las empresas aunque, sin duda, muchas de ellas tienen en alta consideración el capital intelectual que poseen. Sin embargo, esto ya es materia de trabajos desarrollados en Europa y Estados Unidos, desde la década de los años ochenta.

El modelo de medición del capital intelectual que se pretende proponer está basado en las experiencias de otros países, pero el modelo tendrá que adecuarse, debido a nuestro grado de desarrollo en el sector manufacturero. La medición del capital intelectual ofrecerá nuevas formas de comportamiento gerencial a muchos empresarios, de tal forma que permita elevar el valor negociable de la empresa. Asimismo, en la contabilidad de las empresas surgirá una nueva visión de la medición, que el conocimiento de esta nueva valoración producirá en los estados contables, donde los intangibles son y serán, en esta era del conocimiento, lo más importante en toda organización.

Es por ello necesario identificar el modelo que permita la medición del capital intelectual de nuestras empresas manufactureras en el Perú, para valorar la importancia y necesidad de conocer, medir y gestionar aquellos intangibles que no se incluyen en la contabilidad tradicional, pero que están constituyéndose como el principal recurso generador de beneficios que tiene una empresa.

Uno de los principales desafíos, hoy en día en el Perú, será el de establecer modelos para lograr medir la importancia de los intangibles, entendiendo éstos como el conocimiento que poseen las personas o trabajadores, así como su capacidad para aprender, lo que sin duda los convierte en recursos claves para la obtención de beneficios.

Dentro de este marco el problema se formula bajo la siguiente interrogante: ¿Cómo medir el capital intelectual de las empresas de manufactura que faciliten información relevante para la gestión en el seno de la propia empresa y para efectuar el valor negociable y demostrar, así, sus ventajas comparativas y competitivas?.

\section{OBJETIVO GENERAL}

Diseñar un modelo que mida el capital intelectual de las empresas de manufactura para que refleje su valor verdadero y sea éste un intangible importante para la gestión de la empresa o que pueda efectuarse el valor negocia- 
ble de la empresa en el balance general y demostrar, así, las ventajas comparativas y competitivas.

La medición del capital intelectual se justifica por las siguientes razones: Las empresas podrán validar la capacidad de su organización para alcanzar las metas propuestas, obtendrán información necesaria para desarrollar programas de reingeniería, tendrán mayor claridad para invertir en investigación y capacitación especializada de sus trabajadores, promoverán la educación organizacional y podrán calcular el valor real de la empresa, sea para tomarla en consideración dentro del seno de la misma y hacer las modificaciones que consideren o para definir actividades de negociación en el mercado de valores.

Dentro de las limitaciones se puede identificar, entre otras, el difícil acceso a la información dentro de las empresas, la disponibilidad de los indicadores más relevantes y la comprensión de los gerentes, en cuanto al concepto e importancia del Capital Intelectual. Esta limitación ha originado, como consecuencia, la visita a diversas empresas "peruanas exitosas" a las cuales se les ha tenido que explicar la importancia de esta investigación y se está esperando la respuesta de sus pareceres, en tanto se está diseñando el modelo y seleccionando, hipotéticamente, los mejores indicadores para el caso que nos compete.

\section{ANTECEDENTES}

Sveiby (1996) señala que, a mediados de los años ochenta, surge en Suecia la denominada "Comunidad Sueca de Prácticas", resultado de esfuerzos prácticos y de investigación para gestionar las organizaciones del conocimiento y medir el conocimiento. Dentro de ella, se puede diferenciar dos corrientes:

1. Contabilidad de costes para recursos humanos, y

2. "Corriente Konrad", que es el origen del Monitor de Activos Intangibles.

Esta segunda corriente, se inicia con empresas "suecas del conocimiento", que utilizaban indicadores no financieros para controlar y presentar públicamente sus activos intangibles. Posteriormente, estas ideas fueron desarrolladas en la práctica por empresas como WM-data, Skandia y KREAB y se introdujeron en USA y Canadá, gracias al Navegador de Skandia.

Asimismo, el Consejo Sueco para la Industria de Servicios recomienda a las empresas el empleo de determinados indicadores en sus informes anuales indicadores, que son descriptores de su capital humano. En 1994, cuarenta y tres empresas suecas
(43) del conocimiento midieron e informaron de algunos de sus activos intangibles, siguiendo el modelo Konrad (1: Internet).

Además, comenzaron a trabajar sobre el valor real de las empresas que no se muestran en el balance general y esa diferencia la denominaron "Capital Intelectual". Preocupándose de como medirlo, fue así como Leif Edvinsson inició la investigación en la compañía sueca de servicios financieros Skandia en 1995, luego en colaboración con Michael Malone, conocido hombre de negocios, escribieron el primer libro donde explican cómo se calcula el capital intelectual y la utilidad que tiene para las empresas, señalando que el resultado será una transformación revolucionaria de la economía moderna. Al manifestar que el conocimiento y la información son los factores que contribuyen a los procesos de generación de valor en una compañía y que los factores tradicionales de tierra, trabajo y capital producen rendimientos decrecientes, es así que aseveran que el conocimiento y la información se encuentran de manera directa bajo el control de la propia compañía y que deben saberlo, gestionarlo y medirlo (2:18-26).

Algunas empresas europeas han desarrollado sistemas de medida para sus activos intangibles, mostrando los resultados públicamente. Se puede destacar cuatro empresas, todas ellas escandinavas:

1. PLS-Consult (Dinamarca), empresa consultora de gestión,

2. Skandia AFS (Suecia), filial de la empresa de servicios financieros y seguros Skandia,

3. Celemi (Suecia), empresa dedicada al desarrollo y venta de herramientas de formación creativas,

4. WM-data (Suecia), empresa de consultoría y software informático.

Muchas empresas suecas aún no siguen las recomendaciones del Consejo Sueco de la Industria de Servicios por las siguientes razones que se señalan (3: 35-37):

- Las Empresas, en mención, creen que estos informes son inútiles, pues los únicos que dan respuesta a sus informes financieros son los analistas, que ni saben interpretar datos sobre Capital Intelectual, ni tienen tiempo de aprender a hacerlo. Además, los directivos tampoco son conscientes del valor interno de este tipo de informes.

- La existencia de un cierto miedo a que los indicadores revelen demasiada información sobre sus estructuras internas, clientes, competidores, etc.

- La inexistencia de algún modelo teórico riguroso sobre la realización de éste tipo de informes. 
- La falta de experiencia práctica imposibilita tener datos para comparar.

- La ausencia de una robusta evidencia empírica dificulta saber si las medidas son útiles y si de verdad miden aquello para lo que fueron diseñadas.

\section{MARCO CONCEPTUAL}

\section{Capital intelectual}

Mantilla (2000), manifiesta que, en el presente, se entiende por Capital Intelectual al conjunto de sistemas/procesos conformado por el capital humano, el capital estructural y el capital relacional, orientados a la producción y participación de conocimiento, en función de los objetivos estratégicos de la organización (Misión-visión-mercado objetivo).

"Si bien depende en buena parte de la tecnología de la información (Bases de datos relacionales, redes neuronales, inteligencia artificial, etc.) y puede concretarse, a través de formas tangibles (patentes, "copyright", marcas), su naturaleza principal es de carácter intangible, dado que lo último radica en los seres humanos, de manera personal y colectiva. Por lo tanto, el problema principal de las empresas es cómo convertir el conocimiento individual en conocimiento organizacional, lo cual ha dado origen a empresas que aprenden (Aprendizaje organizacional), mediante ingentes esfuerzos por compartir y socializar el conocimiento" (4: 25-28).

Desde la óptica de Edvinson (1997), se hace dos grandes clasificaciones del capital intelectual:

a. Capital Humano: Que corresponde al conjunto de conocimientos, habilidades, actitudes y destrezas de las personas que componen las organizaciones.

b. Capital Estructural: Conocimientos desarrollados y explicitados por las organizaciones, integrado por los siguientes elementos:

- Capital clientes: Activos relacionados con el cliente (Marcas registradas, fidelidad del cliente, listas de clientes, etc.).

- Procesos: Referido a la forma cómo la organización añade valor a través de las diferentes actividades que desarrolla.

- Capacidad de innovación: Entendida como la capacidad de mantener el éxito de la organización, a través del desarrollo de nuevos productos o servicios.

En términos generales, el capital intelectual expresa a los activos (tangibles) menos los activos intangibles, como la capacidad de una compañía para aprender y adaptarse a las nuevas tendencias de la economía de los mercados y de la administración, poniendo énfasis en la gestión del conocimiento, como el acto más significativo de creación de valor (5:18-20).

Edvinson señala que al capital intelectual se le atribuye activos intangibles materializados en las destrezas individuales y conocimientos técnicos, sistemas de información, diseños y marcas de fábricas e, incluso, relaciones con proveedores, clientes y concesionarios.

En EUROFORUM (1998), se señala que "El Capital Intelectual se puede definir como el conjunto de Activos Intangibles de una organización que, pese a no estar reflejados en los estados contables tradicionales genera valor en la actualidad o tiene potencial de generarlo en el futuro" (6: Internet).

Asimismo, Bueno (1998) define al capital intelectual señalando que: "Es la suma y la sinergia de todos los conocimientos que reúne una compañía, toda la experiencia acumulada en sus integrantes, todo lo que ha conseguido en términos de relaciones, procesos, descubrimientos, innovaciones, presencia en el mercado e influencia en la comunidad" (7: 207-208).

Padoveze (2000) presenta las medidas del capital intelectual, bajo los siguientes indicadores (8:4-10):

a. Indicadores para el Capital Humano. Reputación de los empleados de la compañía junto a la empresa de colocación de empleados, años de experiencia en la profesión, tasa de empleados con menos de dos años de experiencia, satisfacción de los empleados, proporción de los empleados, dando nuevas ideas, sugerencias y proporción aplicada, valor añadido por empleado y valor añadido por unidad monetaria de salario.

b. Indicadores para el Capital Estructural. Número de patentes, porcentual de gastos de I \& D (Investigación y Desarrollo) sobre las ventas líquidas, coste de mantenimiento de patentes, costo de proyecto del ciclo de vida por ventas, número de ordenadores individuales conectados al banco de datos, número de veces que el banco de datos es consultado, actualización del banco de datos, contribución al banco de datos, volumen de uso del sistema de información (Sí), costo del "SÍ" por ventas, logro por coste del "Sl", satisfacción con el servicio del "SÍ", tasa de implementación de nuevas ideas por el total de nuevas ideas generadas, número de introducción de nuevos productos, introducción de nuevos productos por emplea- 
do, número de equipos de proyectos multifuncionales, proporción del logro de los nuevos productos introducidos, tendencia del ciclo de vida de los productos en los últimos cinco años, tiempo medio para planificación y desarrollo de producto y valor de las nuevas ideas (Economías y ganancias en dinero).

c. Indicadores para la Clientela y Relaciones. Participación en el mercado, crecimiento en el volumen de negocios, proporción de las ventas por repetición de los clientes, lealtad a la marca, satisfacción de los clientes, reclamación de los clientes, rentabilidad de los productos como una proporción de las ventas, número de alianzas cliente/proveedores y su valor, proporción de los negocios de los clientes o proveedores que los productos y servicios de la empresa representan en valor.

\section{El conocimiento}

El conocimiento, según Padoveze (2000) manifiesta que es "generado y operacionalizado por el ser humano, acumulado y administrado por la sociedad para satisfacción de sus necesidades. Las empresas y las instituciones, que son sociedades de personas con objetivos bien definidos, hacen el papel de reunir y operacionalizar especialidades de conocimiento y con eso consiguen mayor eficiencia y eficacia en la gestión del conocimiento, para atender sus objetivos y cumplir con sus misiones" (9:11-20).

El conocimiento es la base principal de valorización en las organizaciones. Actualmente, en nuestro medio, se ve a las personas como costos y no como ingresos. Pero como se emplee, desenvuelva y organice continuamente el conocimiento dentro de las organizaciones, se transformará en capital intelectual y es, a partir de ese momento, que pasa a agregar valor a los productos o servicios y este capital es, en algunos casos, más valioso que el propio capital físico de la empresa.

Toffler (1981) dice que el momento actual está identificado por la Tercera Ola que es la era del conocimiento caracterizada por el poder del cerebro, en la cual la información asume el papel de principal recurso económico. Hoy, con la sociedad del conocimiento, en los tres factores tradicionales de producción (Recursos naturales, mano de obra y capital), se añade el conocimiento y la inteligencia de las personas, que agregan valor a los productos y servicios.Pasando a representar un importante diferencial competitivo para las empresas que saben adquirirlo, mantenerlo y utilizarlo de forma eficiente y eficaz. Ese conocimiento pasa a generar el capital intelectual que, a su vez, es muy importante, como el capital Económico (10: 29-33).

\section{El capital intelectual y la gestión del conocimiento}

La noción de Gestión del Conocimiento se encuentra estrechamente ligada a la de Capital Intelectual, mientras que el capital intelectual representa los activos intangibles de una empresa (Capital humano, capital estructural y capital relacional), la Gestión del Conocimiento intenta formalizar y sistematizar los procesos de identificación, administración y control del mismo.

Desde esta perspectiva, la Gestión del Conocimiento implica dos variables fundamentales:

a. Los procesos relacionados con la administración del conocimiento generado en la empresa y su estructura de procesos, en términos de cadena de valor

b. Los sistemas de comunicación formal de la organización, así como los sistemas informáticos a través de los cuales la información se almacena, se clasifica y se distribuye a lo largo de toda la estructura de la empresa. Esto implica los aspectos más blandos o menos formalizables de la gestión e incluye los procesos relacionados con la identificación de talentos, formalización de buenas prácticas y estandarización de competencias.

Desde esta perspectiva, la gestión del conocimiento es un proceso de gestión organizacional, cuyo objetivo es identificar el conocimiento que producen los trabajadores talentosos en el desempeño de una empresa para convertirlo en información que pueda ser nuevamente utilizada por el resto de los empleados de esa empresa.

El capital intelectual y la industria manufacturera Según un estudio del Financial and Management Acconting Committee (Técnica Contable), los conceptos básicos relativos a la medida y gestión del capital intelectual se relacionan con tres aspectos (11:101-120).

1. Contexto económico. El crecimiento es mayor en las industrias y naciones que han vuelto a la creación, transformación y capitalización de los conocimientos de lo que es en aquellas que, en sus procesos, están conectadas a la explotación y utilización de recursos naturales. El conocimiento es un diferencial de competitividad.

2. Contexto Contable. La contabilidad tradicional no está habilitada para medir aspectos de la empresa, en cuanto a la capacidad de dirigentes y personal, el valor de las informaciones, de la capacidad tecnológica, potencial de mercado e inversiones en investigación y desarrollo. 
Cuadro 1. Visión en la gestión de la empresa

\begin{tabular}{|c|c|c|}
\hline Aspectos & Visión de la Era Industrial & Visión en la Era del Conocimiento \\
\hline $\begin{array}{l}\text { Percepción del } \\
\text { personal } \\
\text { Flujo de producción }\end{array}$ & Basado sobre procesos & $\begin{array}{l}\text { Conocimiento como generación de } \\
\text { riquezas. } \\
\text { Basado sobre ideas }\end{array}$ \\
\hline $\begin{array}{l}\text { Beneficios / } \\
\text { Inversiones }\end{array}$ & Tendencia de bajada & $\begin{array}{l}\text { Tendencia en aumento por la } \\
\text { creatividad. }\end{array}$ \\
\hline Base de poder & Posición jerárquica & Nivel de conocimiento. \\
\hline Flujo de informaciones & Jerarquía & Redes funcionales y operacionales. \\
\hline
\end{tabular}

Fuente: Baum \& Gonçalves, 2001.

3. Contexto Empresarial. La visión en la gestión de la empresa, a partir de la actual era del conocimiento, con relación a la era industrial, pasa a tener el enfoque que se muestra en el Cuadro 1.

Se encontrarán en desventaja las empresas que no se den cuenta de su capital intelectual. El primer paso para la gerencia de este capital es identificarlo, para después medirlo.

\section{MODELOS DEL CAPITAL INTELECTUAL}

Por un lado, se dice que para la medición del capital intelectual no existen métodos generalmente aceptados y tampoco, sistemas legales. Las mejores prácticas se dan alrededor del análisis de la cadena de valor (Generación y agregación de valor), a través de un conjunto de indicadores cuyo análisis más importante es su desempeño en el tiempo y, sobre todo, el "Benchmarking" (Proceso sistemático y continuo e evaluación de los productos, servicios y procesos de trabajo de las organizaciones) con las mejores prácticas del sector industrial al que se pertenece.

Sin embargo, tanto en estados Unidos como en Europa, diferentes empresas están trabajando sobre la

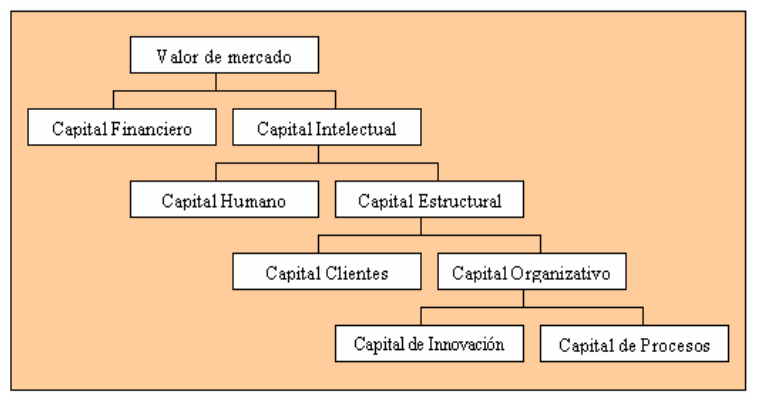

Figura 1. Esquema de valor del mercado de Skandia Fuente: Edvinsson y Malone, 1997. medición del capital intelectual, como el intangible más importante en esta era del conocimiento.

Al respecto, se presentan algunos modelos trabajados y aplicados en esos países por sus correspondientes autores:

\section{Modelo de Skandia}

Edvinsson, en 1991, Skandia AFS contrató a Leif Edvinsson para diseñar una forma de medir el proceso de creación de activos en la empresa. Edvinsson desarrolló una teoría del "Capital Intelectual" que incorpora elementos de Konrad y del «Balanced Score Card» (Kaplan y Norton, 1992, 1993). En los modelos económicos tradicionales, utilizó normalmente sólo el capital financiero, pero la empresa sueca Skandia propone el "Esquema Skandia de Valor", donde se muestra que el capital intelectual está formado por: 1) Capital humano y 2) Capital estructural (12: 73-85, Internet).

\section{Modelo de Edvinsson y Malone}

Este modelo propone una ecuación para calcular el capital intelectual de la empresa, de modo que se puedan realizar comparaciones entre empresas:

Capital Intelectual Organizativo $=\mathrm{i} \times \mathrm{C}, \quad \mathrm{i}=(\mathrm{n} / \mathrm{x})$

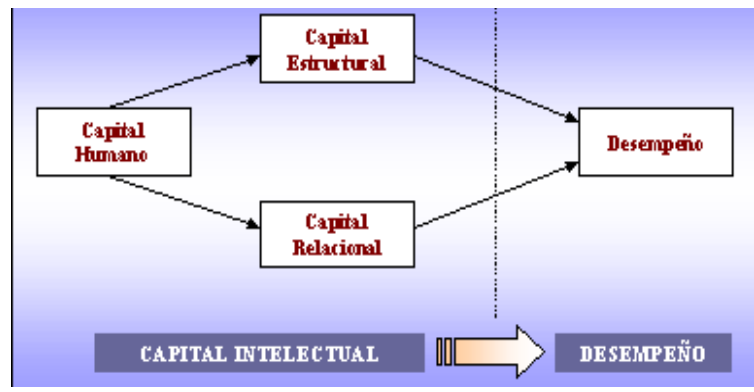

Figura 2. Modelo de la Universidad de West Ontario Fuente: Bontis (1996) en Euroforum, 1998. 


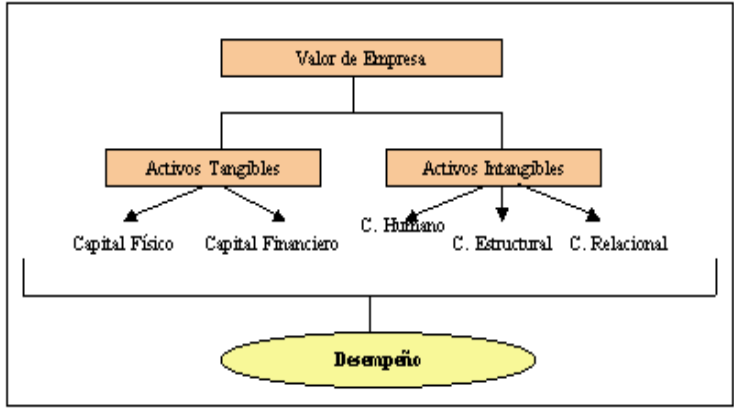

Figura 3. Modelo de medición de capital intelectual Fuente: Euroforum, 1998.

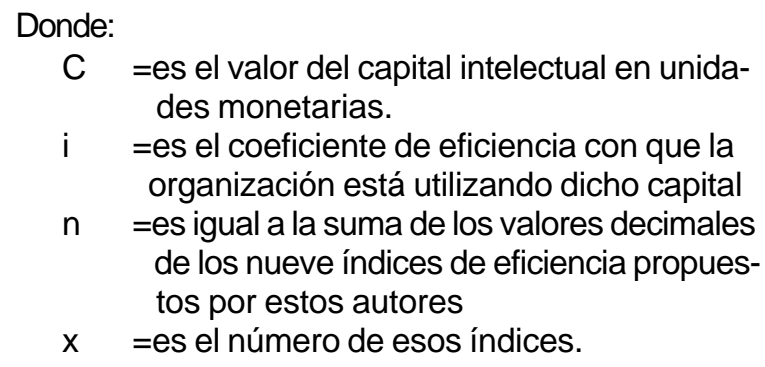

Los elementos de esta ecuación se obtienen a partir de indicadores desarrollados para cada uno de los cinco enfoques propuestos por el Navegador de Skandia.

Modelo de la Unversidad de West Ontario Bontis (1996) estudia las relaciones de "causa-efecto" entre los distintos elementos del capital intelectual y entre éste y los resultados empresariales. Su gran aportación es la comprobación de que el bloque de Capital Humano es el factor explicativo (13: Internet).

\section{Modelo de Euroforum}

La Figura 3 presenta este modelo, allí se puede apreciar el desagregado del valor de la empresa y sus diferentes componentes del capital, tanto físico como intelectual (14: Internet).

\section{AVANCES DEL PROYECTO}

En esta primera parte de la investigación, se trata de interiorizar la concepción y la identificación de los modelos a emplearse, su alcance y primeros ensayos de formulación matemática (ver modelos adjuntos), pues se trata de diseñar un modelo de medición del capital intelectual para las empresas de manufactura, a partir de las experiencias desarrolladas en Norte América y Europa. La propuesta estará referida a encontrar el valor negociable de la empresa, diseñando el modelo que pueda distinguir el valor intangible "CAPITAL INTELECTUAL", para que sumado a los tangibles, dentro de la contabilidad general, se obtenga el "VALOR NEGOCIABLE" de la empresa.

Los objetivos específicos con los que se está iniciando la investigación son:

1. Identificar los tipos de enfoques para la medición del capital intelectual que más semejanza tengan entre empresas manufactureras.

2. Elaborar la estructura de los activos intangibles de las empresas de manufactura que tengan la capacidad de generar valor.

3. Identificar los indicadores comunes de las empresas manufactureras para medir el capital intelectual.

\section{APORTES DE LA INVESTIGACIÓN}

\section{Aporte científico}

El diseño de un modelo que permita con mucha rigurosidad la medición del CAPITAL INTELECTUAL de las empresas manufactureras peruanas, donde se pueda demostrar sus ventajas competitivas.

\section{Aporte social}

Mejor trato y diferentes estímulos a los trabajadores de las empresas peruanas que generen mayor riqueza y, por consiguiente, logren el éxito con sus aportes.

\section{Aporte Económico}

Las empresas y la facultad se verían beneficiadas, las primeras por conocer su situación, en cuanto al capital intelectual que poseen y la facultad por que generaría círculos de trabajo entre los colegas para atender diferentes demandas empresariales en relación al tema.

\section{BIBLIOGRAFÍA}

1. Bontis. (1996). Modelo Intelect: Medición del Capital Intelectual - Euroforum 1998. En: www.fundipe.es. Visitado el 18 abril 2005 (13: Internet).

2. Bueno, E. (1998). El capital intangible como clave estratégica en la competencia actual. Boletín de Estudios Económicos, Vol. LIII. Agosto de 1988, 7: $207-208)$.

3. Edvinsson, L. y Malone, M. (1997). El Capital Intelectual. Grupo Editorial Norma. Barcelona, España. (5: 18-20). 


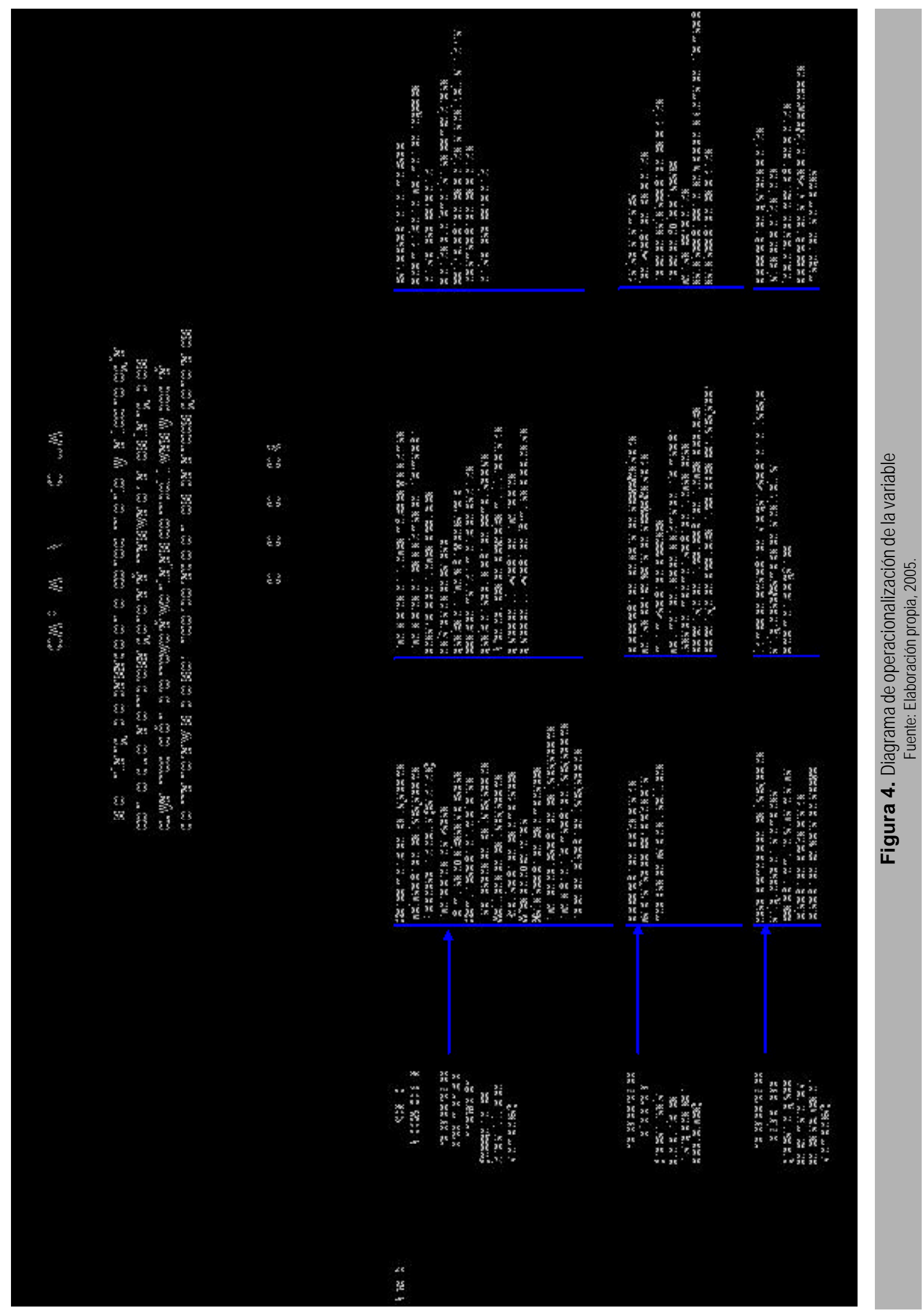




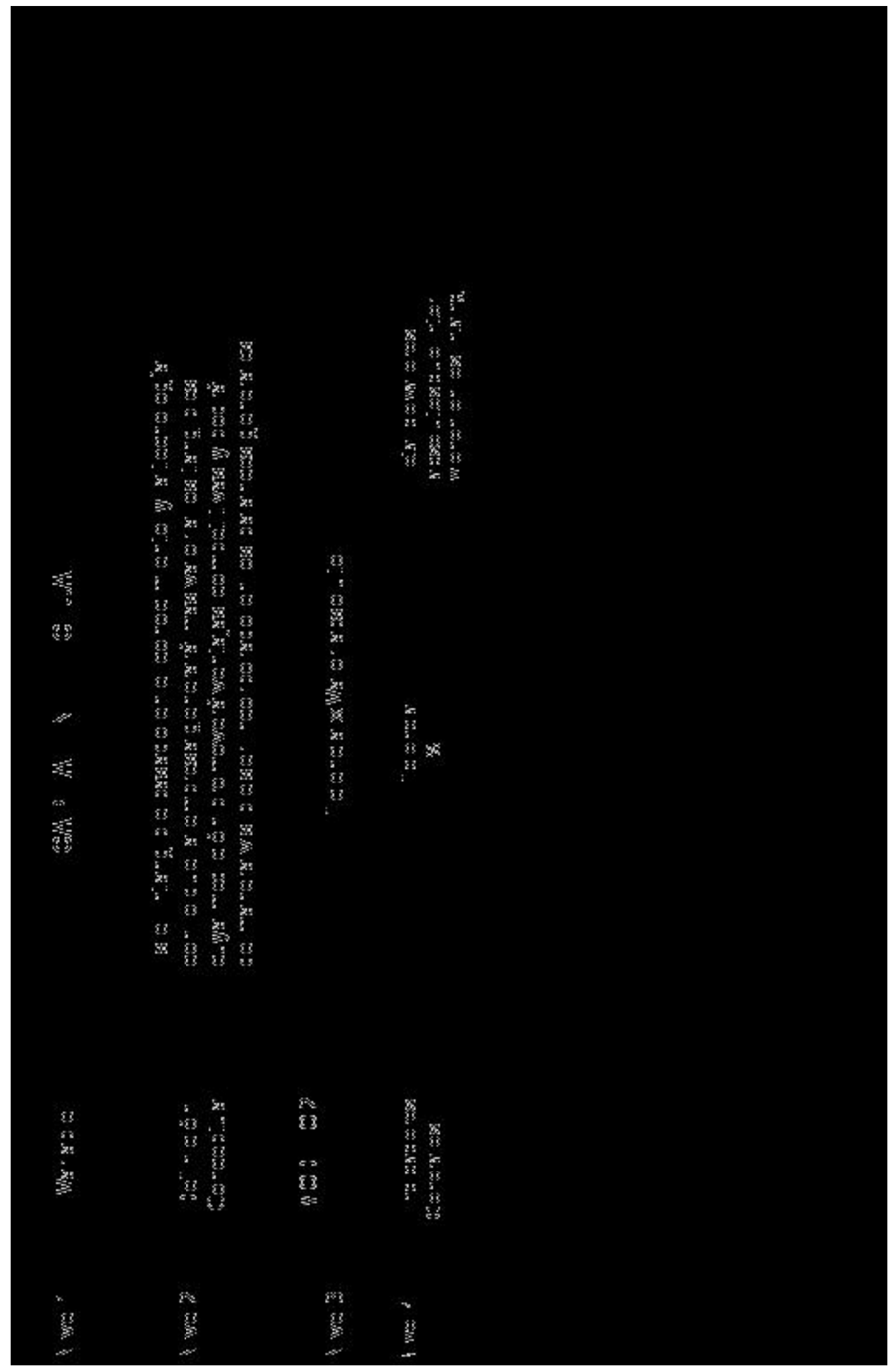


4. EUROFORUM. (1998). Círculos del Conocimiento: El desafío de conservar talento. Fundación lberoamericana del Conocimiento. Barcelona, España. En: www.gestiondelconocimiento.com. Visitado el 20 abril 2005 (6: Internet)

5. Gonçalves, L. y Baum, M. S. (2001). Activos intangibles: Medir o ignorar. Convención de Contabilidad de Río Grande do Sul, 8, Gramado. Anales de la VIII Convención de Contabilidad de Río Grande do Sul. Gramado: RS, 2001. v.3, (11:101120).

6. Mantilla, S.A. (2000). Capital Intelectual: La Contaduría Pública frente a la crisis. Desafíos y propuestas. X Simposio: Contaduría, Universidad de Antioquia. Medellín, Colombia (4:25-28).

7. Padoveze, C.L. (2000). Aspectos de la Gestión Económica del Capital Humano. Revista de Contabili- dad del Consejo Regional de Contabilidad de São Paulo. Año IV, № 14, (8: 4-10), Ibid, (8: 9:11-20).

8. Sveiby, K.E. (1996). The Swedish Community of Practice. Paper for PEI. Conference in Stockholm 25, October "El valor del conocimiento" - Karl E. Sveiby. Revista Mercado, www.mercado.com.ar. Visitado el 12 de febrero 2005 (1: Internet).

9. Sveiby, K.E. (1997). The Intangible Assets Monitor. Journal of Human Resource Costing and Accounting, Vol. 3, No.2, p. (3:35-37).

10.Stewart, T. A. (1998). Capital Intelectual: La Nueva Ventaja Competitiva de las Empresas. Río de Janeiro: Campus, (10:50-51).

11.Toffler, A. (1981). La Tercera Ola. Plaza \& Janes, Impreso y traducido en España, ISBN: 84-0137066-3, (10: 29-33). 